\title{
Experimente zur Raumkognition der Schweiz
}

\section{Kognitive Karten und Kartenkognition}

Jeder Mensch trägt mit sich Vorstellungen über geographische Phänomene und deren räumliche Anordnung herum. Mentale Bilder, die Informationen über räumliche Strukturen und deren Elemente enthalten, werden oft als kognitive Karten (engl. "mental maps») bezeichnet. "Vorstellungskarten» sind seit rund zwanzig Jahren ein Forschungsgegenstand in der amerikanischen Geographie (z. B. LYNCH 1960, GOULD 1966); insbesondere Vertreter der behavioristischen Forschungsansätze sind daran interessiert, da sie die räumlichen Vorstellungsmuster als Einflussgrössen für räumliches Verhalten betrachten. In dieser Tradition ist eine breite Literatur entstanden, die etwa in DOWNS und STEA's «Map in Minds» (1977) repräsentativ zusammengestellt ist. Dieses Werk liegt seit einigen Jahren auch in deutscher Sprache auf (1982) und enthält neben einer Einordnung aus deutscher Sicht spezielle Abbildungsbeispiele und eine ergänzende Bibliographie aus dem deutschen Sprachraum (GEIPEL 1982). "Kognitives Kartieren» wird darin als Handlungsprozess verstanden, der es ermöglicht, Informationen über die räumliche Umwelt zu sammeln, zu ordnen, zu speichern, abzurufen und zu verarbeiten; kognitive Karten sind demnach strukturierte Abbildungen und Vorstellungen eines Menschen über einen Teil der räumlichen Umwelt. Über die Art und Weise, wie diese Raumvorstellungen konkret im Gehirn festgehalten werden, gibt es verschiedene Theorien (vgl. LLOYD 1982). Unbestritten scheint, dass Raumvorstellungen mit in den Problemkomplex geographischer Beschreibung, Analyse und räumlichen Verstehens hineingehören.

Die Thematik der «kognitiven Karten» verbindet die Geographie in zweifacher Beziehung mit der Kartographie. Diese versteht sich als Kommunikationswissenschaft für räumliche Konzepte, ist also interessiert an der Konstruktion und Gestaltung von graphischen Medien, die im Betrachter kognitive Karten auf- oder auszubauen vermögen. Neben dieser Übermittlungsfunktion vom Geographen oder Geowissenschafter zum Informationsbenützer kommt der Kartographie auch eine Rolle bei der Untersuchung der kognitiven Raumbilder zu. Um die gespeicherten Raumstrukturen einer Untersuchung zugänglich zu machen, müssen sie in physische Äquivalente zurückverwandelt werden. Diese physischen Rekonstruktionen der kognitiven Information werden nach moderner Definition durchaus als Karten betrachtet und sind deshalb Objekte kartographischer Untersuchung. Sie lassen Rückschlüsse zu über den allgemeinen räumlichen Wissensstand der Testperson; dies ist dann wiederum ein Anhaltspunkt für die bessere Gestaltung von Karten zur Vermittlung räumlicher Konzepte. Angeregt durch ROBINSON (1952) und durch Forschungsresultate aus der Psychologie hat sich insbesondere in Nordamerika eine Tradition intensiver kartographischer Perzeptions- und Kognitionsforschung entwickelt. Für lange Zeit lag das Schwergewicht auf psychophysischen Untersuchungen, also Fragen, wie verschiedene graphische Dimensionen der Kartensymbole als Stimulus perzeptive Erfahrungen in Auge und Hirn auslösen. Erst in den letzten fünf Jahren sind auf Anregung von oLsoN (1979) vermehrt Kognitionsstudien durchgeführt worden. Der Begriff Kognition umfaßt hier den Gesamtprozeß, durch den ein Lebewesen Wissen über ein Objekt oder die Umwelt erwirbt. Kognition schließt somit neben der Perzeption (Informationserfassung) auch Erkenntnis, Vorstellung, Gedächtnis (Speicherung), Extraktion und Denken (Verarbeitung) mit ein. Es interessiert also, wie der Mensch durch Karten zu neuem räumlichem Wissen und neuen Handlungsstrategien kommen kann. CASTNER (1983) gibt einen Rückblick auf die Forschungsaktivitäten der letzten 30 Jahre, OLSON (1983) versucht die Zielsetzungen für die Zukunft abzustecken, und PETCHENIK (1983) unterzieht diese Forschungsaktivitäten einer kritischen Würdigung.

Zu den Themenbereichen der kognitiven Karten und der Kartenkognition soll hier ein einfaches Experiment vorgestellt werden. Zum einen geht es darum, über räumliches Wissen um schweizerische Lokalitäten mehr zu erfahren, andererseits soll geprüft werden, wie genau dieses Wissen reproduziert werden kann und ob und wie dieser Prozeß durch externe Hilfsmittel (Basiskarten) unterstützt wird. Es geht bei diesem Experiment um ein Erinnern und Einzeichnen von Standorten auf verschiedenen Basiskarten.

Kurt Brassel, Prof. Dr., Daniel Büttler, Andreas Flury, Geographisches Institut der Universität Irchel, Winterthurerstr. 190, 8057 Zürich 


\section{Experimentanordnung}

In der vorliegenden Arbeit soll das Bild der räumlichen Lage einiger Schweizer Orte getestet werden. Die Untersuchung beschränkt sich auf zwölf allgemein bekannte Lokalitäten (Städte, Pässe, Berge, historische Stätten). Die Aufgabe besteht darin, die Lage dieser zwölf Orte ohne Hilfsmittel in vorgelegte Basiskarten einzutragen. Die Auswahl der Orte erfolgte so, daß neben der mentalen Raumkenntnis auch die Beziehung zwischen Einordnungsleistung und speziellen Elementen der Basiskarten getestet werden konnte. Es wurden folgende Stationen gewählt: Gotthard, Erstfeld, Siders, Jungfrau, Rütli, Davos, Bern, Morgarten, Fribourg, Olten, Murten und Glarus. Jede Person wurde aufgefordert, in einer vorgelegten Basiskarte möglichst spontan und ohne Hilfsmittel die Standorte der zwölf Orte mit einem Kreuz zu markieren. Dann wurde die entsprechende Karte wieder eingesammelt und eine neue verteilt. Insgesamt wurden vier Basiskarten verwendet:

a) Umrisse der Schweiz mit Koordinatennetz (Figur 1a)

b) Umrisse der Schweiz mit Gewässernetz und Koordinatennetz (Figur 1b)

c) Umrisse der Schweiz mit Kantonsgrenzen und Koordinatennetz (Figur 1c)

d) Umrisse der Schweiz mit Gewässernetz, Kantonsgrenzen und Koordinatennetz (Figur 1d)

Die Basiskarten lagen im Format DIN A4, also in einem Maßstab von ca. 1 : 1,5 Mio., vor. Instruktionen und Legende sind oben rechts dargestellt. Bei der Aufgabenstellung wurde darauf hingewiesen, $\mathrm{da} ß \mathrm{mit}$ dieser Aufgabe keine individuelle Leistungsbeurteilung verbunden sei. Das Experiment wurde mit insgesamt 159 Versuchspersonen durchgeführt, davon waren 69 Geographiestudenten des 3. Semesters (Universität Zürich), 38 Mittelschüler der 2. Gymnasialklassen (Kantonsschule Oerlikon) und 52 Absolventen einer Maturitätsschule für Erwachsene (Akademikergemeinschaft Zürich). Im folgenden werden wir die drei Gruppen vereinfacht als Studenten, AkadAbsolventen und Kantonsschüler bezeichnen.

Anhand der Kreuzsymbole der insgesamt 630 Karten (6 wurden nicht abgegeben) wurden anschließend in einer auf jedem Blatt vorgedruckten Datenzeile die Koordinaten verschlüsselt (auf Kilometer genau) und digital gespeichert. Alsdann wurden die Ergebnisse quantitativ ausgewertet.

\section{Auswertungsstrategie}

Die insgesamt 7218 Beobachtungen (159 Personen x 4 Karten x 12 Lokalitäten) können nun nach folgenden Kriterien ausgewertet werden:
Fig. 1a-d: Basiskarten für die Einordnung der Orte: a) Umrisse der Schweiz, b) Umrisse + Gewässer, c) Umrisse + Kantonsgrenzen, d) Umrisse + Gewässer + Kantonsgrenzen. Originalbasiskarten je im Maßstab DIN A4.

\section{Den Basiskarten beigefügte Legende:}

\section{Instruktionen für den Einordnungstest}

Tragen Sie die in der Tabelle aufgeführten 12 Lokalitäten möglichst spontan als kleines Kreuz in die Karte ein.

Wichtig: Setzen Sie neben jedes Kreuz die entsprechende Lokalitätsnummer.

1. Gotthard

2. Erstfeld

3. Siders

4. Jungfrau

5. Rütli

6. Davos

7. Bern

8. Morgarten

9. Fribourg

10. Olten

11. Murten

12. Glarus

- Anteil der fehlenden Eintragungen pro Lokalität. Dieser Index gibt ein Maß für den Bekanntheitsgrad einer Lokalität.

- Mittlere Lage der geschätzten Punktlokalitäten, absolut oder in Relation zur "wahren» Lage. Zur Definition der mittleren Lage kann das arithmetische Mittelzentrum oder das Medianzentrum (s. BAHRENBERG und GIESE 1975, S. 33ff.) beigezogen werden. Als Index für die Güte der Punktlokalisation kann nun die Distanz zwischen dem arithmetischen Mittelzentrum und der wahren Position verwendet werden. Dieses Maß gibt an, wie gut die kognitive Karte mit der realen Raumgeometrie übereinstimmt (mittlere Lageverschiebung). Man kann dafür argumentieren, daß Orte, die extrem weit von ihrem wahren Standort eingetragen sind, eigentlich als «unbekannt» eingestuft werden könnten. Da es aber problematisch ist, einen willkürlichen Grenzwert einzuführen (z. B. 50 oder $100 \mathrm{~km}$ ), haben wir für unsere Untersuchungen darauf verzichtet.

- Zweidimensionale Streuungsparameter: Als Maße können die Standardabweichungen einer Population von ihrem Mittelwert in beiden Dimensionen des Koordinatensystems verwendet werden. Besser geeignet ist die sog. Standarddistanz, die der Wurzel aus dem Mittel der quadrierten Distanzen zwischen Beobachtungspunkten und arithmetischem Mittelzentrum entspricht (s. BAHRENBERG und GIESE 1975, S. 49-50). Die Standarddistanz als Streuungsgröße ist ein Maß für die «Unsicherheib» bzw. das «NichtÜbereinstimmen» der verschiedenen Versuchsteil- 



un Fig. 1b
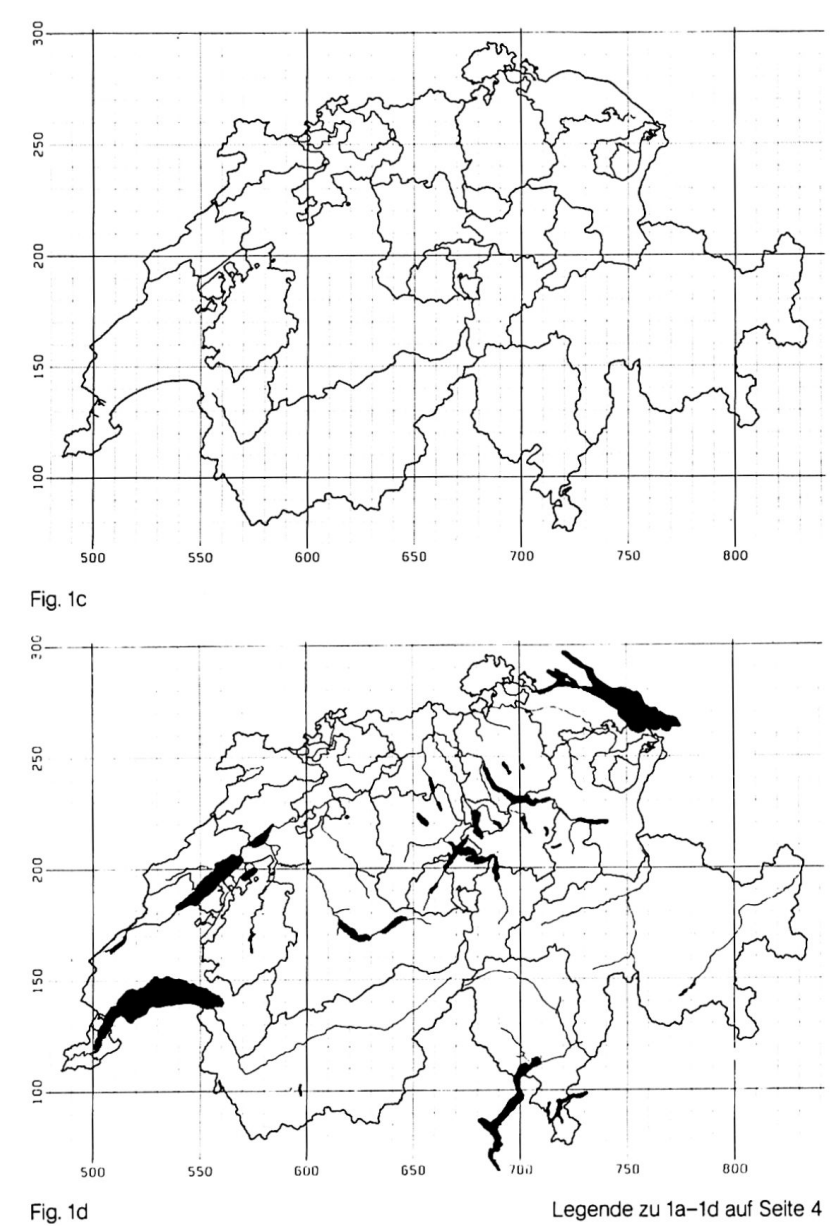
nehmer. Dieses $\mathrm{Ma} ß$ ist nicht notwendigerweise korreliert mit dem Abstand des Mittelzentrums vom wahren Wert, d.h. eine Gruppe von Personen kann mit großer "Sicherheit» (kleine Streuung) einen Punkt gut oder schlecht (kleiner oder großer Abstand vom wahren Wert) plazieren oder umgekehrt.

Die Standarddistanz beschreibt eine mittlere räumliche Streuung, berücksichtigt also die spezielle räumliche (Winkel-)Ausrichtung nicht. Um zusätzliche Aussagen über die Strukturen der Antworten zu ermöglichen, verwenden wir hier die "Standardabweichungsellipsen» (EBDON 1977, S. 113-118). Es handelt sich um Ellipsen, die statistisch rund 45\% aller beobachteten Punkte einer Population in sich schließen. Die große Achse der Ellipse fällt mit der Regressionsgeraden durch die Punkteschar zusammen, die kleine Achse liegt senkrecht dazu. Die Längen der beiden Halbachsen entsprechen den Standardabweichungen in den entsprechenden Richtungen.

Die Standardabweichungsellipsen drücken wiederum die «Unsicherheit» der Gruppe aus. Im Gegensatz zur Standarddistanz wird diese Größe hier räumlich differenziert (winkelabhängig) dargestellt.

- Mittel der Abstände zwischen den einzelnen Beobachtungspunkten und dem wahren Wert. Dieses Maß vereinigt Aspekte der mittleren Lageverschiebung und der Punktestreuung. In der räumlichen Statistik nur selten gebraucht, stellt es eine pragmatische und leicht einfühlbare Größe dar.

Die genannten statistischen Maße (Zahl der Antworten, Abstand zwischen Mittelzentrum und wahrem Wert, Standarddistanz, Abweichungsellipsen, Mittel der Distanzen zum wahren Wert) können nun für verschiedene (Teil-)Gruppierungen des Gesamtdatenbestandes berechnet werden. Die Auswertung kann folgendermaßen erfolgen:

A Gesamtdatei

B Differenziert nach vier Variablen:

a) 12 Lokalitäten

b) 4 Basiskarten

c) 3 Klassen (Studenten, Akad-Absolventen, Kantonsschüler)

d) Reihenfolge der Kartenpräsentation

C Bivariate Kombinationen:

Da die vier oben genannten Variablen nicht linear zusammenhängen, ist es sinnvoll, für die Auswertung auch bivariate Kombinationen zu berücksichtigen:
ab) Lokalitäten x Basiskarten
ac) Lokalitäten $x$ Klassen
ad) Lokalitäten $x$ Reihenfolge
bc) Basiskarten $x$ Klassen
bd) Basiskarten $x$ Reihenfolge
cd) Klassen $x$ Reihenfolge

D Schließlich wäre eine differenzierte Auswertung der 3er- und 4er-Kombinationen möglich:

abc) Lokalitäten x Basiskarten x Klassen

acd) Lokalitäten $x$ Klassen $x$ Reihenfolge

bcd) Basiskarten $x$ Klassen $\times$ Reihenfolge

Wir werden hier das gesammelte Datenmaterial nicht erschöpfend auswerten, sondern beschränken uns auf die Darstellung von Zusammenhängen von speziellem Interesse. Die volle Auswertung ist bei FLURY et. al (1986) nachzulesen. Wir bedienen uns dabei der explorativen Datendarstellung in Form von digitalen Karten und Diagrammen, die die Zusammenhänge aufzeigen. Zur Erhärtung einiger Hypothesen werden statistische Tests verwendet.

\section{Resultate}

Fehlende Eintragungen: Von den insgesamt 7560 einzutragenden Stationen fehlten $342(4,5 \%)$. Die Ausfallquote ist bei den Studenten 4,2\%, bei den Berufsmittelschülern und Kantonsschülern rund 4,8\%. Schlecht bekannt waren vor allem die Lokalitäten Erstfeld und Morgarten (rund 11,5\%) sowie Siders $(6,5 \%)$ und Rütli (5,9\%). Fehlende Eintragungen waren bei der Karte a am häufigsten $(5,8 \%)$, bei der Karte $d$ am seltensten $(4,1 \%)$.

Abweichungen vom wahren Wert: Im Mittel liegen die geschätzten Orte $22,5 \mathrm{~km}$ (oder $14,6 \mathrm{~mm}$ auf den vorgelegten Karten) vom wahren Standort entfernt. Insgesamt zeigen die Abweichungen leicht nach Westen. (Die Verschiebung nach Westen 1,7 km bzw. $1,1 \mathrm{~mm}$ ist signifikant; die Verschiebung nach Norden $0,1 \mathrm{~km}$ bzw. $0,06 \mathrm{~mm}$ ist nicht signifikant.)

Betrachten wir das Mittel aller Standardabweichungen, so beträgt diese in Ost-West-Richtung $17 \mathrm{~km}(11 \mathrm{~mm})$, in Nord-Süd-Richtung $11,5 \mathrm{~km}(7,5 \mathrm{~mm})$; die Differenz ist statistisch gesichert. Insgesamt streuen also die Punkte in der geographischen Länge mehr als in der Breite. Dies hängt möglicherweise mit der Gesamtform der Schweiz oder lokalen Bedingungen der gewählten Orte zusammen.

Eine Differenzierung nach Lokalitäten ist aus der Figur 2 ersichtlich. Hier sind die wahren Standorte mit Kreuzen angegeben, und eine Gerade verbindet sie mit den arithmetischen Mittelzentren aller Schätzungen. Um diese Zentren sind Abweichungsellipsen gezeichnet. Aus Gründen der Übersicht sind diese mit Achsen halber Länge dargestellt worden, enthalten somit statistisch nur rund $14 \%$ der Beobachtungswerte. Die Standardabweichungsellipsen zeigen die Charakteristiken der Punktanordnung: je peripherer die Kreuze bezüglich der Ellipsenzentren liegen, um so signifikanter ist der Abstand des Mittelzentrums vom wahren Wert. Alle Verschiebungen sind 


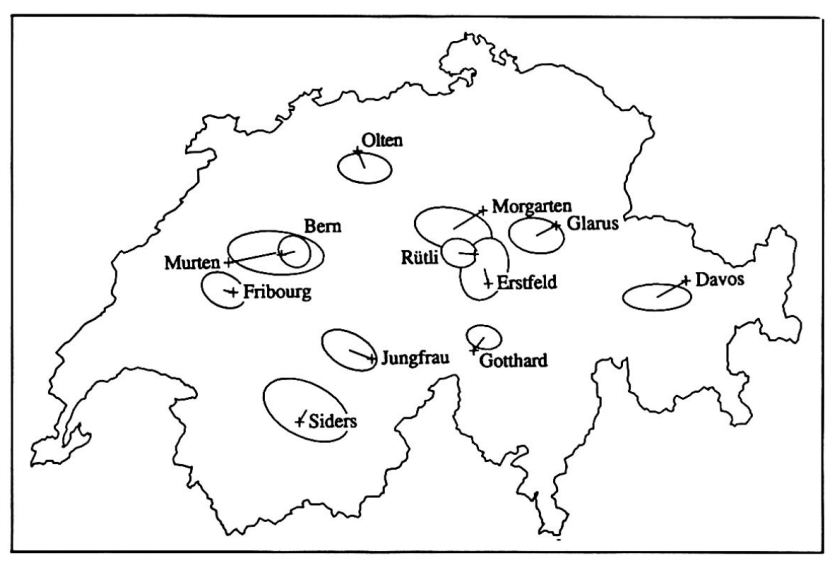

Fig. 2



Wahre Lage der Orte (Kreuze); Standardabweichungsellipsen mit halbem Radius, angeordnet um das arithmetische Mittelzentrum; Distanz wahre Lage-Mittelzentrum (Gerade).

Figur 2: Darstellung der Resultate der Gesamtstichprobe:

Figur 3: Darstellung der Resultate anhand der Basiskarte a (Umrisse der Schweiz)

Figur 4: Darstellung der Resultate anhand der Basiskarte (Umrisse + Gewässer + Kantonsgrenzen)

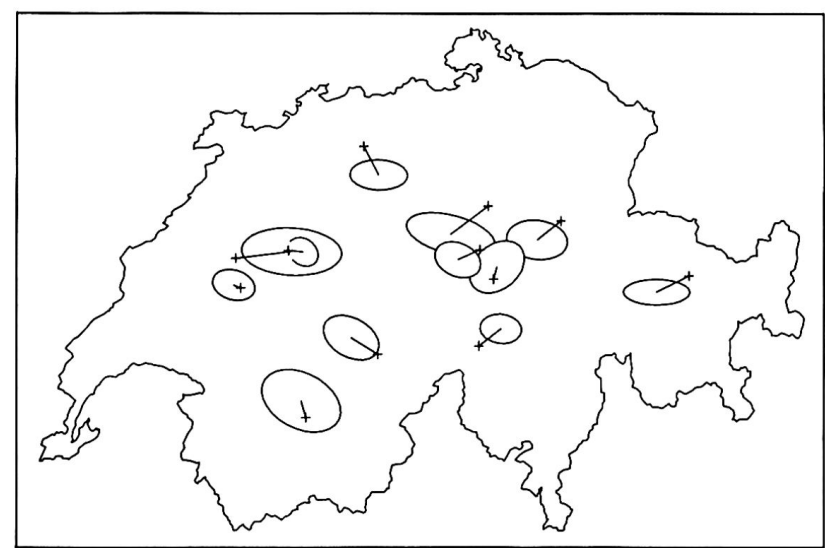

Fig. 4 
statistisch gesichert. Wir überlassen es dem Leser, die individuellen Verschiebungen zu interpretieren, möchten aber darauf hinweisen, daß ein Zusammenhang zwischen Streuung und Distanz zur nächstgelegenen Referenzinformation der Basiskarten bestehen mag. Auch fällt auf, daß in den meisten Fällen eine Verschiebung von der Landesgrenze zum Zentrum stattgefunden hat. Ferner steht fest, daß die Verzerrungsellipsen unterschiedliche Größe und Richtung aufweisen. Kleinste Unsicherheit herrscht bei der Lokalisation des Gotthard, von Bern und Rütli, größte bei Siders, Morgarten und Murten. Bei der Mehrzahl der Orte überwiegt die Ost-West-Komponente der Ellipsen, einzige Ausnahme bildet Erstfeld.

Eine differenzierte Interpretation ist möglich, wenn neben den Lokalitäten zusätzlich nach den vier Basiskarten unterschieden wird. Die mittleren Abstände vom wahren Wert betragen für die Basiskarte a (nur Schweizergrenzen) im Mittel 26,1 km (17 mm), für die Karten b (Gewässer) 22,3 km (4,5 mm), c (Kantonsgrenzen) 21,3 km (13,8 mm) und d (Gewässer + Kantonsgrenzen) 20,4 km (13,3 mm). Das Mittel für Karte a unterscheidet sich signifikant von den anderen Gruppen; b und c können statistisch nicht getrennt werden. Und auch die Resultate der Karte d sind nicht signifikant besser als b und c gemeinsam. Diese Zusammenhänge gelten auch für die Mittel der Standarddistanzen (Flächen der Ellipsen). Die Interaktionen zwischen Lokalitäten und Basiskarten werden aus den Figuren 3 und 4 sichtbar. Diese beiden Karten zeigen die Resultate der Basiskarte a (Schweizergrenze, Figur 3) und der Basiskarte d (Schweizergrenzen + Gewässer + Kantonsgrenzen, Figur 4). Aus Raumgründen können hier die Resultate der beiden anderen Basiskarten nicht gezeigt werden. Zusätzliche Basiselemente reduzieren in der Regel sowohl die mittleren Abstände zum wahren Wert als auch die Streuung der Schätzungen. Die Abstände der Mittelzentren zum wahren Wert (Distanz zwischen Ellipsenzentrum und Kreuz) sind in Figur 4 für alle Stationen außer Fribourg und Erstfeld kleiner als in Figur 3; die Ellipsenfläche reduziert sich bei rund der Hälfte der Stationen, bei der anderen Hälfte bleibt sie gleich. Es läßt sich zeigen, daß bei Bern, Fribourg und Erstfeld das Gewässernetz die Einordnung erschwert. Im Falle von Erstfeld bewirkt es, daß die Abweichungsellipse stärker nach Nord-Süd gedreht wird, ohne sie zu verkleinern. Die Zusammenhänge zwischen Fehlergröße, Lokalitäten und Basiskarte werden ferner illustriert in Figur 5. Die Orte sind so angeordnet, daß die Station mit minimaler Fehlersumme (Bern) vorn, der Ort mit größtem Fehler (Murten) rechts hinten liegt; die vier Grundlagenkarten sind von links nach rechts angeordnet. Wie dies zu erwarten ist, fällt die Oberfläche von hinten nach vorn ab. Auffällige Ausnahmen sind die hohen Werte für Fribourg, Siders und Bern in der Gewässerspalte (Bern liegt auf dem Koordinatenschnittpunkt 600/200, der auf allen
Karten eingezeichnet ist; bei Vorliegen des Gewässernetzes scheint man diese Tatsache oft zu vergessen). Murten und Morgarten sind auf der Leerkarte (a) besonders schwer einzuordnen. Aus Figur 5 wird auch sichtbar, daß bei einigen Orten das Gewässernetz, bei anderen die Kantonsgrenzen die Einordnung erleichtern. Sicher spielt dabei die Nähe zu einem identifizierbaren Kennzeichen auf der Basiskarte eine Rolle. Bei Davos und Erstfeld verbessern weder die Kantonsgrenzen noch das Gewässernetz die Einordnung.

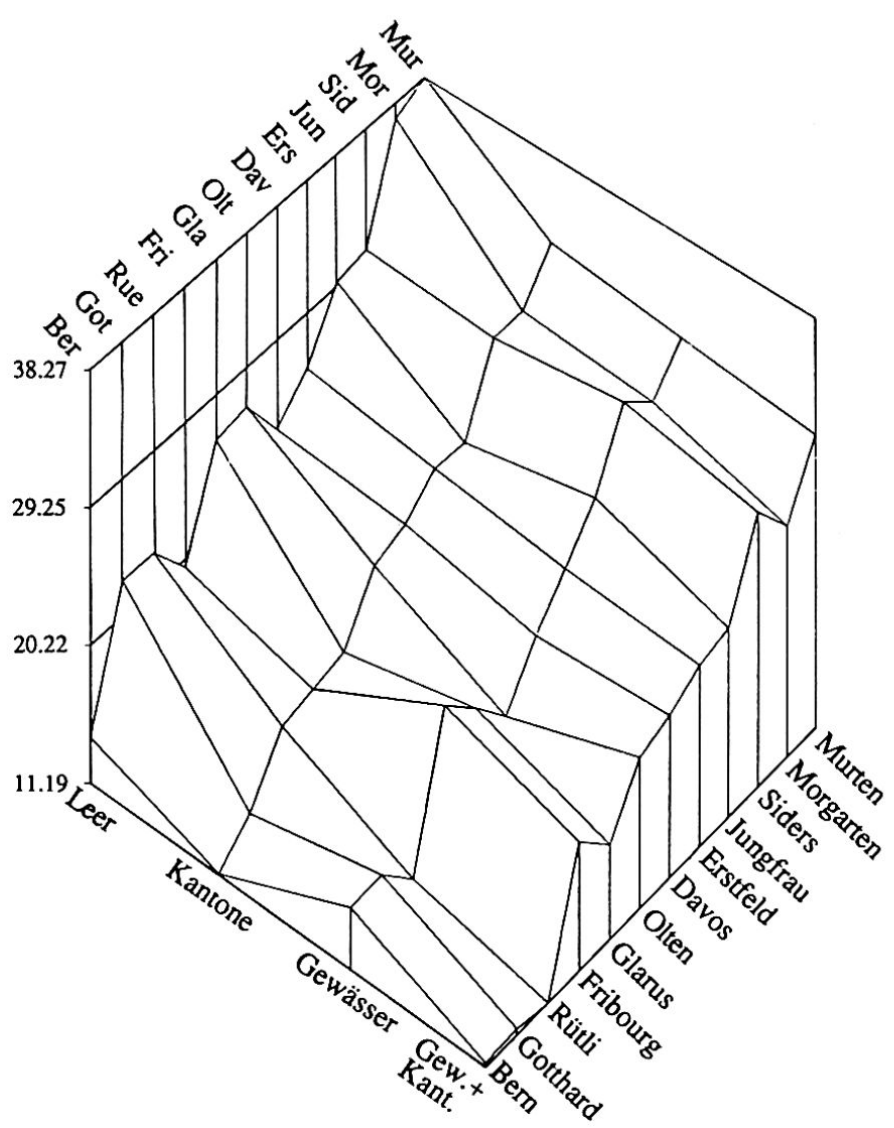

Figur 5: Mittlerer Abstand vom wahren Wert als Funktion von Lokalitäten und Basiskarten.

Als letztes möchten wir die Differenzierung nach Schulklassen miteinbeziehen (Figuren 6 und 7). Insgesamt sind die Resultate der Studenten (Mittel der Abstände vom wahren Wert $17,4 \mathrm{~km}$ bzw. $11,3 \mathrm{~mm}$ ) besser als die der Akad-Absolventen (19,9 km bzw. $12,9 \mathrm{~mm}$ ) und insbesondere die der Kantonsschüler $(35,5 \mathrm{~km}$ bzw. $23,1 \mathrm{~mm})$. Letztere hatten vor allem mehr Mühe, Murten, Siders und Erstfeld einzuordnen; die Studenten waren weniger vertraut mit Morgarten. Die Relation zwischen Klassen und Basiskarten sind in Figur 7 gezeigt. Die Abweichungen der Kantonsschüler sind am größten bei allen vier Basiskarten, diejenigen der Akad-Absolventen größer 


\begin{tabular}{|l|ll|ll|}
\hline \multicolumn{4}{|l|}{ Extremwerte der Mittel der Abstände vom wahren Wert } \\
\hline & Minimum & & \\
\hline nach Lokalïäten & Bern & $13 \mathrm{~km}(8,45 \mathrm{~mm})$ & Murten & $33,1 \mathrm{~km}(21,5 \mathrm{~mm})$ \\
nach Klassen & Studenten & $17,4 \mathrm{~km}(11,3 \mathrm{~mm})$ & Kantonsschüler & $35,5 \mathrm{~km}(23,1 \mathrm{~mm})$ \\
nach Basiskarten & Karte d & $20,4 \mathrm{~km}(13,3 \mathrm{~mm})$ & Karte a & $25,0 \mathrm{~km}(16,25 \mathrm{~mm})$ \\
\hline
\end{tabular}

Tabelle 1

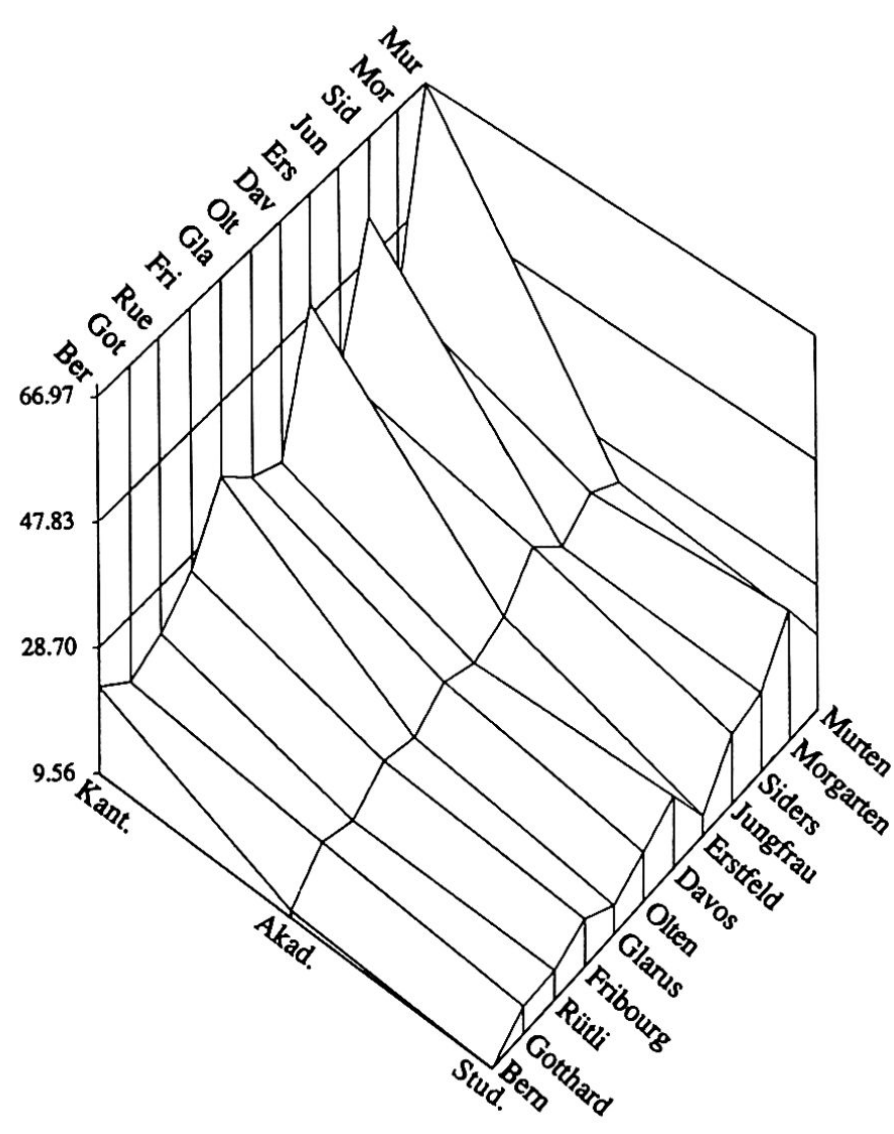

Figur 6: Mittlerer Abstand vom wahren Wert als Funktion von Lokalitäten und Klassen.

als die der Studenten. Bei allen Gruppen wird die Leistung durch ein erstes Basiskartenelement signifikant, durch ein zusätzliches aber nur unwesentlich verbessert.

Vergleichen wir zum Schluß die drei Variablen Lokalitäten, Basiskarten und Klassen, so lassen sich folgende Feststellungen machen (Tabelle 1): Die Abweichungen (Mittel der Abstände vom wahren Wert) variieren am meisten zwischen den Lokalitäten, d. h. der Bekanntheitsgrad eines Ortes ist das entscheidende Kriterium für die Einordnungsleistung. Als zweites Kriterium ist die Auswahl der Befragungsteilnehmer (Klassen) anzuführen, was wiederum mit

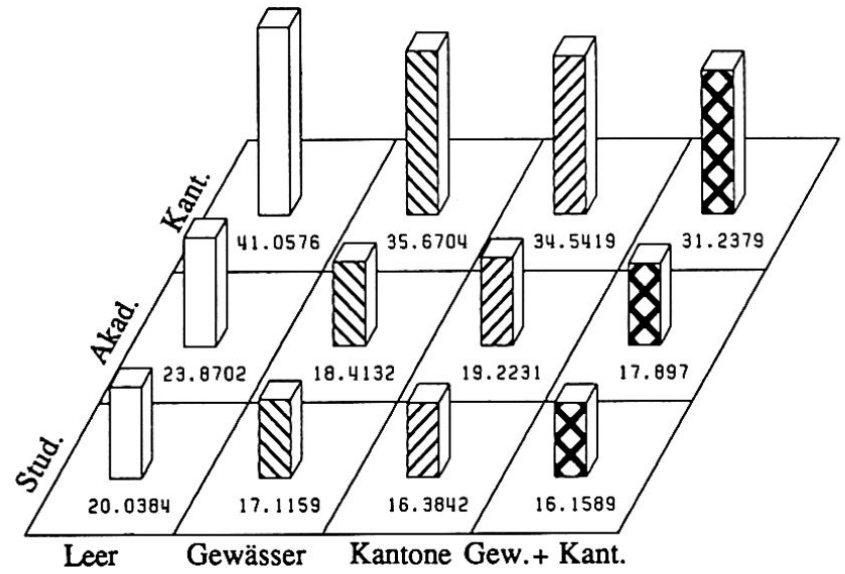

Figur 7: Mittlerer Abstand vom wahren Wert als Funktion von Klassen und Basiskarten.

dem Kenntnisstand räumlicher Phänomene in Zusammenhang gebracht werden kann. Erst in dritter Linie spielt die verwendete Basiskarte eine Rolle. Zusätzliche Basisinformation bringt jedoch bei allen Versuchsgruppen eine Verbesserung der Lokalisierungsleistung, in der Regel auch bei allen Lokalitäten.

\section{Ausblick}

In einem empirischen Experiment haben wir versucht, Aussagen über kognitive Karten verschiedener Personengruppen zu machen. Die zwölf gefragten Orte waren eineın überwiegenden Teil der Befragten bekannt, ihr Standort ist aber einer systematischen Verschiebung unterworfen. Die Richtung dieser Verschiebung variiert zwar von Ort zu Ort, doch ist ein Trend vom Kartenrand zum Zentrum hin zu verzeichnen. Die Einordnungsleistung variiert sowohl nach Lokalitäten als auch nach Personengruppen. Flußnetz oder Kantonsgrenzen unterstützen die Lokalisierung, hingegen bringt eine Kumulierung dieser Basisdaten nur geringe Verbesserungen. Diese Studie konnte 
erste Beziehungen aufzeigen und Hypothesen formulieren. Den Zusammenhängen zwischen Einordnungsleistung und Nachbarschaft zu Basiskartenelementen muß weiter nachgegangen werden. Auch sollten weitere Bevölkerungskreise (Berufstätige, Betagte) miteinbezogen werden. Weiterhin steht offen, ob befragte Personen mit Wohnsitzen in anderen Teilen der Schweiz andere Antwortmuster liefern. Aus technischen Gründen hat sich die vorliegende Arbeit auf die Einordnung von Punktlokalitäten beschränkt, doch können in zukünftigen Arbeiten wohl lineare und flächenhafte Objekte mitberücksichtigt werden. Kartenkognition ist ein weites Feld für zukünftige Forschungsaktivitäten.

\section{Verdankungen}

Frau M. Wegmann und Dr. K. Kistler haben sich freundlicherweise bereit erklärt, mit ihren Schulklassen an diesem Experiment teilzunehmen. Herr U. Helbling und Frau Chr.Karrer haben bei der Codierung der Daten mitgewirkt, Herr M. Steinmann hat die Figuren aufgearbeitet. Die Autoren bedanken sich bestens für diese Unterstützung.

\section{Literatur}

ARNHEIM, R. (1976): The Perception of Maps. In: The American Cartographer, Vol. 3 Nr. 1, 5-1.

BAHRENBERG, G., GIESE, E. (1975): Statistische Methoden und ihre Anwendung in der Geographie. Stuttgart: Teubner.

CASTNER, H.W. (1983): Research Questions and Cartographic Design. In: Progress in Contemporary Cartography, Vol.2, Taylor F. D. R. (ed.), 37-68, Chichester: Wiley \& Sons.

DOWNS, R. M., STEA, D. (1973): Cognitive Mapping and Spatial Behaviour. In: Image and Environment, 8-26, Chicago: Aldine Publishing Company.

DOWNS, R. M., STEA, D. ed. (1977): Maps in Minds. New York: Harper \& Row Publishers. Deutsche Ausgabe (1982): Kognitive Karten: Die Welt in unseren Köpfen. New York: Harper \& Row Publishers.
EBDON, D. (1977): Statistics in Geography, a Practical Approach. Oxford: Basil Blackwell.

FLURY, A., BUETTLER, D., BRASSEL, K.E. (1986): Untersuchungen zur Raumkognition der Schweiz. Geoprocessing Reihe Vol. 6, Geogr. Institut Universität Zürich (in Vorb.).

GEIPEL, R. (1982): Kognitives Kartieren als Bindeglied zwischen Psychologie und Geographie (Einführung des Herausgebers). In Downs \& Stea: Kognitive Karten. New York: Harper \& Row Publishers, 7-14.

GOULD, P. (1966): On Mental Maps. Michigan: Inter-University Community of Mathematical Geographers, Vol. 9.

GOULD, P., WHITE, J. R. (1974): Mental Maps. London: Penguin Books.

GILMARTIN, P.P. (1981): The Interface of Cognitive and Psychophysical Research in Cartography. In: Cartographica, Vol. 18 Nr. 3, 9-20.

KISHIMOTO, H. (1975): "Mental Maps" und Kartographie. In: Vermessung, Photogrammetrie, Kulturtechnik, Vol.1 Nr.3, 62-64.

LLOYD, R. (1982): A Look at Images. In: Annals of Assoc. of American Geogr., Vol. 72 Nr. 4, 532-548.

LYNCH, K. (1960): The Image of the City. Cambridge, Mass.: MIT Press.

OLSON, J. M. (1979): Cognitive Cartographic Experimentation. In: The Canadian Geographer, Vol. 16 Nr. 1, 34-44.

OLSON, J.M. (1983): Future Research Directions in Cartographic Communications Designs. In: Progress in Contemporary Cartography, Vol. 2, Taylor F.D. R. (ed.), 257-284. Chichester: Wiley \& Sons.

PETCHENIK, B. B. (1983): A Map Maker's Perspective on Map Design Research 1950-1980. In: Progress in Contemporary Cartography, Vol. 2, Taylor F.D.R. (ed.), 37-68. Chichester: Wiley \& Sons.

ROBINSON, A.H. (1952): The Look of Maps. Madison: University of Wisconsin Press.

ROBINSON, A.H., PETCHENIK, B.B. (1976): The Nature of Maps. Chicago/London: The University of Chicago Press.

TUAN, Y.F. (1975): Images and Mental Maps. In: Annals of the Assoc. of American Geographers. Vol. 65 Nr. 2, 205-213.

TVERSKY, B. (1981): Distortions in Memory of Maps. In: Cognitive Psychology, Vol. 13, 407-433.

VANSELOW, G.W. (1974): Mental Maps in Cartography. In: Canadian Cartographer, Vol. 11, Nr. 2, 190-191. 